\title{
The relationship between self-traumatized and self-vulnerable automatic associations and posttraumatic stress symptoms among adults who have experienced a distressing life event
}

\author{
Simon E. Blackwell ${ }^{1}$ (D) Thomas Ehring $^{2}$ (D) Thomas E. Gladwin $^{3}$ (D) . Jürgen Margraf ${ }^{1}$ (D) Marcella L. Woud ${ }^{1}$ (D)
}

Accepted: 26 October 2021

(c) The Author(s) 2021

\begin{abstract}
Convergent evidence supports a crucial role for dysfunctional appraisals in the development and maintenance of posttraumatic stress disorder (PTSD). However, most research in this area has used self-report measures, assessing only explicit forms of such negative cognitions; the relevance of their more automatically-activated counterparts, as assumed by cognitive models, remains relatively unexplored. The current study aimed to further our understanding of the potential utility of measuring automatic dysfunctional associations in the context of posttraumatic stress. The relationship between scores on two different implicit association tests (IATs) and posttraumatic stress symptoms was investigated in a sample of adults $(N=279)$ who reported having experienced a potentially traumatic negative life event. Participants completed the two IATs (one assessing self-traumatized associations, the other self-vulnerable associations), a self-report measure of dysfunctional appraisals, and measures of posttraumatic stress symptoms and other aspects of psychopathology online. Scores indicating higher levels of dysfunctional associations on both IATs were associated with higher levels of posttraumatic stress symptoms. Only scores on the IAT measuring self-vulnerable associations, and not the IAT measuring self-traumatized associations, continued to show an association with posttraumatic stress symptoms after controlling for explicit dysfunctional appraisals. Overall, the results indicate the value of investigating PTSD-relevant automatic associations to further develop our understanding of cognitive processes implicated in posttraumatic stress.
\end{abstract}

Keywords Implicit association test · Post-traumatic stress disorder · Appraisal · Dysfunctional cognitions · Cognitive vulnerability

Although many people will experience a traumatic event in their lifetime, for example $60.7 \%$ of men $51.2 \%$ of women in the US National Comorbidity survey (Kessler et al., 1995), the extent to which this causes them lasting distress varies greatly: Some people will experience no impact beyond the short-term, while at the other end of the spectrum others will experience prolonged distress and functional impairment. One potentially severe outcome following a trauma

Simon E. Blackwell

simon.blackwell@rub.de

1 Mental Health Research and Treatment Center, Faculty of Psychology, Ruhr-Universität Bochum, Massenbergstrasse 9-13, 44787 Bochum, Germany

2 Department of Psychology, LMU, Munich, Germany

3 Centre for Mental Health, Institute for Lifecourse Development, The University of Greenwich, London, UK is post-traumatic stress disorder (PTSD), which is characterised by symptoms such as involuntary memories of the event, avoidance of reminders, and hyperarousal (American Psychiatric Association, 2013). The lifetime prevalence rate of PTSD in the US National Comorbidity survey was estimated at $7.8 \%$ (Kessler et al., 1995). Converging evidence from cross-sectional, prospective, experimental, and treatment research indicates a pivotal role for cognitive processes, in particular dysfunctional appraisals, in the development and maintenance of post-traumatic distress and PTSD, with the implication that these appraisals are important targets for prevention and treatment (Brown et al., 2019; McNally \& Woud, 2019; Woud et al., 2019b).

According to several cognitive models of PTSD (e.g., Brewin et al., 1996; Dalgleish, 2004; Ehlers \& Clark, 2000; Foa, Steketee, \& Rothbaum, 1989), if an individual tends to appraise the traumatic event in a dysfunctional manner, then this can contribute to the experience of 
post-traumatic distress and potentially to the development and maintenance of PTSD. Such dysfunctional appraisals may concern oneself, for example the implications of one's reactions to the trauma (e.g. "I am a weak person"), or other people (e.g. "People can't be trusted). These appraisals will tend to fuel not only distress and a sense of current threat, but also dysfunctional coping strategies that maintain symptoms such as intrusive memories and avoidance (Ehlers \& Clark, 2000). The models' predictions are supported by a large body of empirical evidence, with both cross-sectional and prospective studies tending to support the idea that negative appraisals are a risk factor for higher levels of PTSD symptoms and for the development and maintenance of PTSD (e.g., Bryant \& Guthrie, 2007; Ehring et al., 2008; Kleim et al., 2012; VossbeckElsebusch et al., 2014).

Experimental analogue studies also support the hypothesised causal role of dysfunctional appraisals in post-traumatic stress, in that the induction of a negative, as opposed to positive, appraisal style is associated with higher levels of analogue post-traumatic stress symptoms (e.g., Woud et al., 2012, 2018). Similarly, an RCT amongst inpatients with PTSD found that reducing dysfunctional appraisals directly via computerized training was associated with reductions in PTSD symptoms (Woud et al., 2021). Finally, there is evidence that decreases in negative appraisals may play a role in reducing PTSD symptoms during standard psychological therapy, in that changes in appraisals from session to session have been found to predict reductions in PTSD symptoms but not vice-versa (Kleim et al., 2013). The importance of measuring appraisals in the context of trauma is highlighted by the inclusion of negative cognitions about oneself in the DSM-5 definition of PTSD (American Psychiatric Association, 2013).

One open question in relation to the dysfunctional appraisals observed PTSD is the extent to which these are the product of cognitive processes that occur relatively automatically and rapidly, outside of direct conscious control, or rather involve conscious reflection. Cognitive theories of psychopathology often discriminate between automatic and reflective processes (for a review, see Teachman et al., 2019), although such a distinction has come under criticism (e.g., Gladwin \& Figner, 2014; Keren \& Schul, 2009). This automatic vs. reflective conceptualization has its roots in in early cognitive theories of emotional psychopathology (e.g., Beck \& Clark, 1997). To illustrate, it has been proposed that anxious individuals process potentially threat-related cues in a selective manner, and that this processing bias occurs quickly and automatically, without requiring any conscious reflection (for a review of information processing biases in emotional psychopathology, see Mathews \& MacLeod, 2005; and in the context of PTSD, see Vasterling \& Hall, 2019; Woud et al., 2017).
In the context of dysfunctional trauma appraisals, the automatic/reflective distinction has indeed informed cognitive models of PTSD (e.g., Brewin et al., 1996; Ehlers \& Clark, 2000). However, the utility and relevance of such a distinction in this domain is not yet clear. Studies investigating dysfunctional appraisals in the context of PTSD have tended to measure these via self-report measures. However, if dysfunctional appraisals also have an automatic, uncontrollable component, it should be possible to measure this automatic activation, and there are several reasons why this is important. From a theoretical perspective, reflective and automatic appraisals may be differentially related to different aspects or symptoms of PTSD. It may even be that being able to measure automatic activation of dysfunctional appraisals would provide predictive or explanatory power in accounting for post-traumatic distress, beyond that provided by assessing only that aspect of dysfunctional appraisals available for conscious reporting. Further, if automatic appraisals do play an important role in PTSD, then they would be relevant to measure in a treatment context as a potential indicator of treatment success or risk of relapse: While there are effective treatments for PTSD, such as trauma-focussed cognitive behaviour therapy (National Institute for Health and Clinical Excellence, 2018), there is still considerable room for improvement in terms of outcomes and relapse rates. Identification and measurement of automatically activated cognitions could contribute a missing piece to the broader puzzle of assessing PTSD treatment outcomes. For example, successful reduction of explicit but not automatic dysfunctional cognitions in response to a treatment could indicate a risk factor for relapse in need of further attention.

There is some evidence to support the potential utility of measuring relatively automatic dysfunctional cognitions in the context of PTSD from studies that have used a variant of the implicit association test (IAT; Greenwald et al., 1998). The IAT aims to measure the associative strength in memory between two concepts or constructs, indexed via a performance (reaction time) measure. Participants use two response keys to sort stimuli (e.g. words) into four categories, two representing a target concept of interest and its control (e.g. me vs. other), and two representing opposite poles of the attribute of interest (e.g. traumatized vs. healthy). Over the course of the IAT, each target category is paired with each attribute, and the difference in reactions times between when a congruent target-attribute pair (e.g. me and traumatized) share a response key and when an incongruent target-attribute pair (e.g. me and healthy) share a response key is interpreted as providing an indirect measure of the strength of the target-attribute associations. The IAT has been extensively used across many areas of emotional psychopathology (e.g., Roefs et al., 2011; Teachman et al., 2019), and many domains of psychology more broadly (Greenwald et al., 2020). 
In the context of PTSD, Engelhard et al. (2007) administered an IAT measuring self-vulnerable associations to a sample of Dutch soldiers before and after deployment to Iraq. The only relationship they found between scores on this IAT and PTSD symptoms were at 5-months post-deployment, where higher scores (i.e. stronger self-vulnerable associations) were associated with greater PTSD symptom severity, including when controlling for self-reported vulnerability and neuroticism. The authors suggest stronger self-vulnerable associations may therefore reflect a consequence of PTSD symptoms, as IAT scores did not predict future PTSD symptom severity. The content of this IAT could potentially be seen as relating to the sense of being permanently changed for the worse as a result of the trauma, which is an important component of cognitive models of PTSD (Ehlers et al., 2000; Ehlers \& Clark, 2000). Lindgren et al. (2013) further tested two different IATs in a sample of adults visiting an online research portal who reported having experienced a traumatic event. One IAT assessed the association between self as traumatized vs. healthy, and the other assessed the association between remembering as dangerous vs. safe. Scores on the self-traumatized IAT, but not the remembering-dangerous IAT, were related to severity of PTSD symptoms. In a follow-up study with a similar sample, scores on this IAT were found to explain additional variance in PTSD symptom severity even when including self-report negative appraisals (measured using the subscale of the PTCI asking about the self) in a regression. The content of this self-traumatized IAT could be seen as mapping on to one of the key aspects of the model of Ehlers and Clark (2000), that is negative appraisals of the trauma, its consequences, and the experience of posttraumatic stress symptoms itself. The results of these studies suggest the potential value of assessing automatically activated dysfunctional trauma cognitions in seeking to understand the importance of negative cognitive processes in PTSD (for additional studies using the IAT in the context of posttraumatic stress, see e.g., Bluemke et al., 2017; Bockers et al., 2016; Rüsch et al., 2011; Woud et al., 2021).

The current study aimed to build on and extend the previous work using IATs to measure PTSD-relevant automatic associations, by deploying both IATs identified as having potential utility as mentioned above (self-vulnerable and self-traumatized) together in one study. In addition to testing conceptual replication of the previous results in a different sample, this would extend this research by further exploring the relationship between these different kinds of trauma-relevant dysfunctional associations and posttraumatic stress. The two IATs were administered online to a sample of adults who reported having experienced a potentially traumatic negative life event, and who additionally completed measures of explicit dysfunctional cognitions (the PTCI), posttraumatic stress symptoms, and other aspects of psychopathology.
We hypothesised that higher scores on the IATs (indicating stronger dysfunctional associations) would be associated with higher levels of posttraumatic stress symptoms. Additionally, we hypothesised that scores on the IAT would explain unique variance in such symptoms including when controlling for explicit self-report dysfunctional appraisals and other potential predictors of PTSD symptom severity.

\section{Methods}

\section{Participants and Recruitment}

Participants were recruited via PsyWeb (https://psyweb.unimuenster.de/), a non-commercial online panel for individuals from the general population who are interested in taking part in psychological research. Inclusion criteria were being aged 18 or over, and reporting having experienced a distressing negative life event (cf. Woud et al., 2019a). A total of 518 participants provided consent, and of these 420 provided details of a distressing or traumatic life event. A further 342 completed the first part of the study (both IATs and details of trauma), with 281 completing the rest of the survey (all questionnaires). One participant wrote in a free text box that they had not taken part seriously, and another provided an invalid value for their age (5 years old), and so their data was removed. All remaining participants were included in the analyses with no further exclusions. This left a final data set of 279 participants $(189,67.74 \%$, female). No additional inclusion/ exclusion criteria were applied based on participants' patterns of responding on the IAT. A post-hoc power analysis using $G *$ Power 3.1.9.2 (Faul et al., 2007) indicated that our sample size would provide $80 \%$ power to detect fairly small effect sizes $\left(\mathrm{f}^{2}>0.026\right)$ for single regression coefficients in multiple regressions of the type conducted in this study at $p<.05$. No financial incentives or other compensation were provided for participation.

\section{Design and Procedure}

The study was cross-sectional and conducted online in German. Pre-registration was completed on AsPredicted.org (registration, \#10252, see https://aspredicted.org/jy3dp. pdf). The Supplementary Materials contain further detailed information about the study methods.

\section{Materials}

Online Platforms The first section of the study (informed consent, details of the negative life event, the two IATs) was administered via a custom-built online platform implemented using Java Server Pages and JavaScript on a secure server at Ruhr-Universität Bochum. The second section of 
the study (demographic information and standardised questionnaire measures) was implemented in Unipark (http:// www.unipark.com/). Source code for the IAT online platform is available at https://osf.io/qsyu6/.

\section{Details of Negative Life Event and Trauma History Checklist} (THC; Holmes \& Steel, 2004) Participants were first asked if they had ever experienced a distressing or traumatic life event, and if they selected 'no', they were taken to a 'thank you' page and did not continue with the study. Participants who selected 'yes' were asked to describe the event in a few words or sentences, then categorise it into one of 12 categories (or a 13th category of 'other' with a free text box to provide details; see Supplementary Materials) via an adapted version of the Trauma History Checklist originally developed by Holmes and Steel (2004) and used in previous similar studies (e.g., Woud et al., 2018). Finally, participants provided details of how long in the past the event occurred (in years and months), rated how distressing the event was for them at the time, and how distressing the event was for them now (on a 10-point scale from 1 to 10, anchored at either end with Not distressing at all and Extremely distressing).

Implicit Association Tests (IAT; Greenwald et al., 1998) Two Implicit Association Tests (IAT) were used, a Self-Traumatized IAT and a Self-Vulnerable IAT (details provided below). Participants completed these one after the other, with the order counterbalanced across participants (alternating order). The IATs were introduced with a general brief introduction explaining that we intended to measure participants' reaction times to categorise words. Participants were asked to react as quickly as possible, but to slow down if they were making mistakes. Participants used the keys ' $\mathrm{A}$ ' and ' $\mathrm{L}$ ' to respond. Both IATs had a standard structure of seven blocks: Attribute practice (e.g. 'traumatized' vs. 'healthy'; 20 trials); Target practice (e.g. 'self' vs. 'other'; 20 trials); Combined (congruent) attribute and target practice (20 trials); Combined (congruent) attribute and target block (40 trials); Target reversed practice (20 trials); Combined (incongruent) attribute and target (reversed) practice (20 trials); Combined (incongruent) attribute and target (reversed) block (40 trials). All participants had the same initial target/attribute-key assignment, in that the non-dysfunctional attribute and 'other' target were always initially on the right hand side, and thus the congruent combined blocks always preceded the incongruent combined blocks (see individual IAT descriptions for definitions of congruent/incongruent trials). If participants provided an incorrect response, they saw a red cross in the centre of the screen and the task only continued once they had made the correct response.

Self-Traumatized IAT. The Self-Traumatized IAT was translated and adapted from the 'traumatized self'
IAT of Lindgren et al. (2013), using the same translated stimuli and task structure as Woud et al. (2018). ${ }^{1}$ This IAT aimed to assess the associative strength between the target concepts 'self versus others' and the attributes 'traumatized versus healthy'. Word stimuli were as follows: Self: self, me, my, mine (German: selbst, ich, mein, mir); Others: not me, other, they, them (German: nicht ich, andere, sie, ihnen); Traumatized: traumatized, damaged, broken, distressed (German: traumatisiert, verletzt, zerbrochen, belastet); Healthy: healthy, adjusted, capable, whole (German: gesund, angepasst, kompetent, ganz). In congruent combined blocks, words belonging to the categories trauma and self shared a response key and words belonging to the categories healthy and not me shared a response key. In incongruent combined blocks, words belonging to the categories trauma and not me shared a response key and words belonging to the categories healthy and me shared a response key. Participants who appraised their self as relatively more traumatized than others should therefore have faster RTs in the congruent trauma \& me (and healthy \& not me) assignments compared to the incongruent trauma \& not me (and healthy \& me) assignments. Split-half reliability in the current study was $0.72,95 \%$ CIs: $[0.65,0.78]$.

Self-Vulnerable IAT. The Self-Vulnerable IAT was translated and adapted from that used by Engelhard et al. (2007). This IAT aimed to assess the associative strength between the target concepts 'self versus others' and the attributes 'vulnerable versus invulnerable. Word stimuli were as follows: Self: self, me, my, mine (German: selbst, ich, mein, mir); Others: not me, other, they, them (German: nicht ich, andere, sie, ihnen); Vulnerable: vulnerable, powerless, weak, helpless (German: verwundbar, kraftlos, schwach, hilflos); Invulnerable: invulnerable, powerful, strong, resilient (German: unverwundbar, kraftvoll, stark, belastbar). In congruent combined blocks, words belonging to the categories vulnerable and self shared a response key, and words belonging to the categories invulnerable and others shared a response key. In incongruent combined blocks, words belonging to the categories invulnerable and self shared a response key, and words belonging to the categories vulnerable and others shared a response key. Participants who appraised their self as relatively more vulnerable than others should therefore have faster RTs in the congruent vulnerable \& me (and invulnerable \& not me) assignments compared to the incongruent vulnerable \& not me (and invulnerable \& me) assignments. Split-half reliability in the current study was $0.64,95 \%$ CIs: $[0.55,0.72]$.

\footnotetext{
$\overline{1}$ with one change: the use of the word belastet instead of erschüttert
} 
Post-Traumatic Cognitions Inventory (PTCl; Foa et al., 1999; German Translation: Ehlers, 1999; German Validation: Müller et al., 2010) The PTCI comprises 33 statements that reflect potential negative appraisals of a traumatic experience. Twenty-one items are negative appraisals about the self (e.g. I am a weak person), seven are negative appraisals about the world (e.g., People can't be trusted), and five reflect self-blame (e.g., The event happened because of the way I acted). As in previous similar studies (e.g., Woud et al., 2018, 2019a), the instructions were adapted such that participants were instructed to rate the statements according to the thoughts they had experienced during the past month about the negative event they had listed at the beginning of the study. Statements were rated on a scale from 1 (totally disagree) to 7 (totally agree), and the individual item scores summed to produce a total or subscale score. Following Lindgren et al. (2013), in this study we focussed primarily on the Self subscale, measuring the clearest explicit counterpart to the automatic self-related dysfunctional appraisals thought to be measured by the IATs. Internal consistency (Cronbach's alpha) in the current study was $0.96,95 \%$ CIs: $[0.95,0.96]$ for the total scale, $0.95[0.94,0.96]$ for the Self subscale, $0.85[0.81,0.88]$ for the Self-blame subscale, and $0.90[0.88,0.92]$ for the World subscale.

\section{Posttraumatic Stress Disorder Checklist for DSM-5 (PCL-5; Ger-} man Version: Krüger-Gottschalk et al., 2017) The PCL-5 is a 20-item self-report questionnaire assessing symptoms of PTSD according to DSM-5. As in previous similar studies (e.g., Woud et al., 2018, 2019a), participants were ask to rate the symptoms in relation to the negative life event listed at the beginning of the study, and for their experience over the past month. Ratings were made on a scale from 0 (not at all) to 4 (extremely). Cronbach's alpha in the current study was 0.94 [0.93, 0.95].

\section{Depression Anxiety Stress Scales - Short Form (DASS-21;} German Version: Nilges \& Essau, 2015) The 21-item short form of the DASS assesses the experience of symptoms of depression, anxiety, and stress over the past week, using seven items per subscale. Ratings are made on a scale from 0 (never) to 3 (almost always). Cronbach's alphas were 0.93 $[0.91,0.94]$ for the depression subscale, $0.82[0.78,0.85]$ for the anxiety subscale, and $0.90[0.89,0.92]$ for the stress subscale.

\section{State-Trait Anxiety Inventory (STAI; German Version: Laux} et al., 1983) The STAI contains two 20-item measures of anxiety, one reflecting state (i.e. right now) levels of anxiety, and one trait (i.e. in general) levels. Statements are rated on a scale from 1 (not at all / almost never) to 4 (very much so / almost always), with a small number of these reverse-scored. Cronbach's alphas in the current study were $0.95[0.94,0.96]$ for the trait scale and $0.95[0.94,0.96]$ for the state scale.

\section{Procedure}

After providing informed consent, participants provided details of their chosen negative life event, followed by the two IATs. A weblink then took them to the second part of the survey, where they completed demographic information, the PTCI, PCL-5, DASS-21, and STAI (in this order). ${ }^{2}$ They were then debriefed and thanked for taking part then directed to the PsyWeb website. See Fig. 1 for a diagrammatic representation of the procedure.

\section{Statistical Analyses}

Data from the separate parts of the study (negative life event, IATs, questionnaires) were aggregated into an SPSS (IBM SPSS Statistics for Windows, Version 25.0. Armonk, NY: IBM Corp.) file, with subsequent analyses conducted in RStudio (RStudio, Inc, 2016).

IATs were scored using the D600 method (Greenwald et al., 2003). First, reaction times $>10,000 \mathrm{~ms}$ were removed. All trials from practice congruent, congruent, practice incongruent, and incongruent blocks were used in scoring, with a $600 \mathrm{~ms}$ penalty added to incorrect responses. A d-score was calculated separately for the practice and full-length blocks by subtracting the mean RT for congruent trials from the mean RT for the incongruent trials and dividing this difference score by the standard deviation of the RT for the relevant blocks. The final IAT score was the mean of these two d-scores, such that more positive scores reflected faster performance in the congruent than in the incongruent blocks (i.e. more dysfunctional associations).

Prior to testing our main hypothesis, we conducted preliminary analyses of the reliability and validity of the IATs as indirect measures of dysfunctional traumarelevant appraisals. We examined their internal consistencies, correlations with each other, and relationship with self-report self-relevant dysfunctional appraisals as measured by the PTCI Self subscale. To test our hypothesis about the associations between the IATs and trauma symptoms on the PCL-5, a hierarchical regression approach was used. First, scores on the two IATs

\footnotetext{
$\overline{2}$ The study also included the Life Orientation Test - Revised (LOTR; German version: Glaesmer, Hoyer, Klotsche, \& Herzberg, 2008), completed after the STAI. On a final webpage for the study, participants received feedback about their LOT-R score, in terms of the approximate percentile of the population into which their score fell. The inclusion of such feedback on an aspect of the study is a requirement of the PsyWeb panel, and we chose the LOT-R score for this purpose as it seemed a measure that would be of interest and intuitive to understand for participants, but did not provide any information (e.g. symptoms) that could be misinterpreted for self-diagnostic purposes. The LOT-R data is included in the publically available database for this study, but we do not analyse or consider it further here.
} 


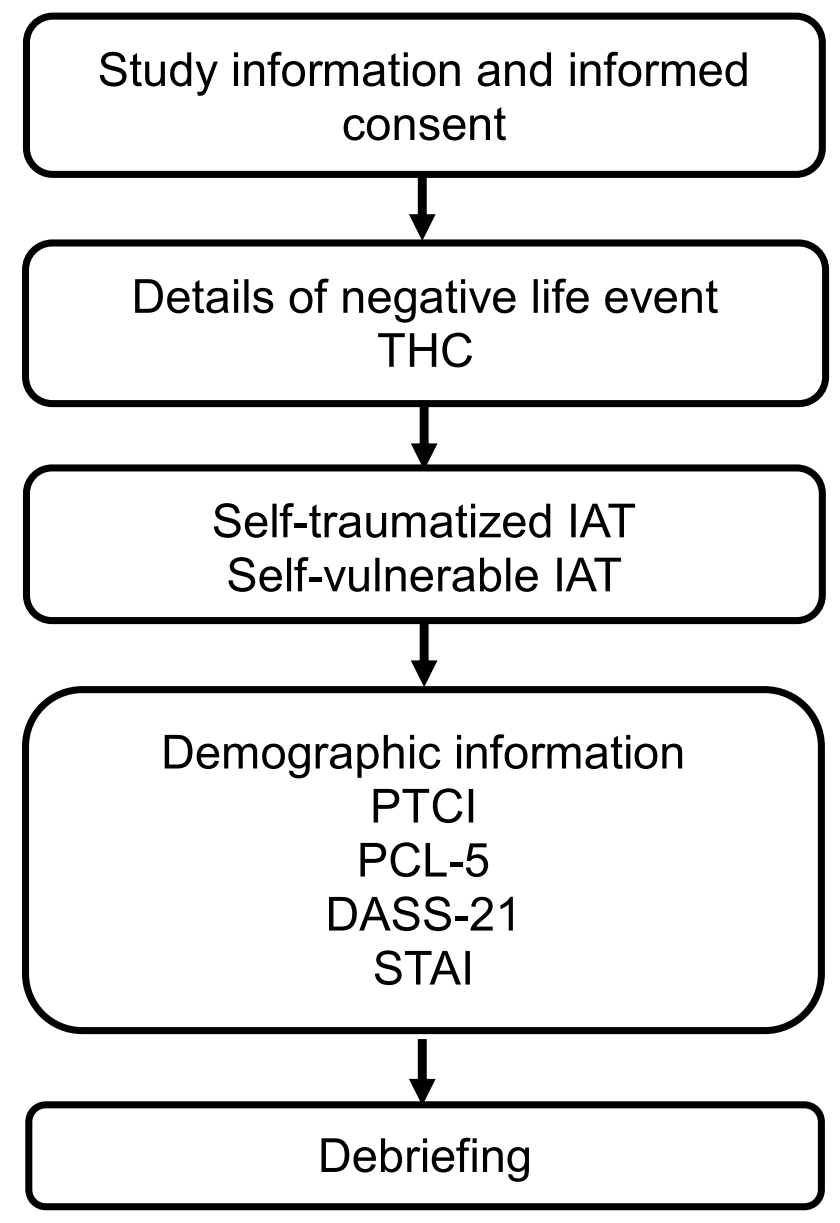

Fig. 1 Study Procedure. Note. Order of the two IATs was counterbalanced across participants. THC=Trauma History Checklist; IAT =Implicit Association Test $;$ PTCI=Post-Traumatic Cognitions Inventory; $P C L-5=$ Posttraumatic Stress Disorder Checklist for DSM-5; DASS-21 = Depression Anxiety Stress Scales - Short Form; STAI = State-Trait Anxiety Inventory

were included as predictors. In a next step, scores on the PTCI Self subscale were added to test whether scores on the IATs explained any variance in posttraumatic stress symptoms above that explained by self-report dysfunctional appraisals of the self. A final step was used to control for other variables potentially relevant in predicting PTSD symptom severity. To select these variables we followed Lindgren et al. (2013), who controlled for gender and a measure of trauma exposure, operationalised as number of events checked on the THC. In our study, participants could only check one event on the THC (the most distressing), and as a measure of severity of trauma exposure we used participants' rating of distress at the time of the event. The assumptions underlying the regression were checked following the steps outlined in Field (pp. 288-301, 2012), and robustness of the results further checked via calculation of 10,000 bootstrapped 95\% confidence intervals for the regression coefficients. More details are provided in the supplementary material, and the full regression models and diagnostics, as well as scripts use to score the IAT, are available via the scripts on the Open Science Framework at https://osf.io/qsyu6/.

\section{Results}

\section{Participants}

Table 1 shows participant characteristics and correlations of the different measures with the two IATs. Further correlations are shown in Supplementary Table 1.

\section{Trauma Severity and Characteristics}

The most commonly endorsed trauma category on the THC was 'other' $(n=155,55.56 \%)$, followed by life-threatening illness $(n=43,15.41 \%)$, car accident, sexual assault, or non-sexual assault by an acquaintance or family member ( $n=15,5.38 \%$ for each), then serious accident/fire/ explosion $(n=13,4.66 \%)$. All other categories were endorsed by fewer than 10 participants. Examples of 'other' traumatic events provided were the death of a relative or loved one, or witnessing a serious accident happening to someone else. Table 1 shows other characteristics of the reported traumatic events. Notably, there was great variation in how long ago the event happened, with the median being 11.67 years.

\section{Relationship between IAT Scores and Self-Reported Dysfunctional Appraisals}

Scores on the two IATs correlated strongly with each other and with the PTCI (see Table 1). There was not a statistically significant difference between the two IATs' split-half reliabilities, $\mathrm{X}^{2}(1)=1.89, p=.17$. A regression with PTCISelf score as dependent variable, and scores on both IATs as independent variables, showed that both IATs predicted unique variance in PTCI-Self score: Self-Traumatized IAT: $B=27.39,95 \%$ CIs [17.58, 36.49], $S E=4.43 \beta=0.37,95 \%$ CIs [0.25, 0.49], $S E=0.06, t=6.18, p<.01$; Self-Vulnerable IAT: $B=10.81,95 \%$ CIs [2.28, 19.93], $S E=4.47 \beta=0.15$, 95\% CIs [0.03, 0.26], $S E=0.06, t=2.42, p=.02$; overall model adjusted $\mathrm{R}^{2}=0.21$.

\section{Relationship between IAT Scores and Post-Traumatic Stress Symptoms}

A regression with PCL score as dependent variable, and scores on both IATs as independent variables, showed 
Table 1 Participant characteristics

\begin{tabular}{llll}
\hline & Mean (SD, Range) & $\begin{array}{l}\text { Correlation with Self- } \\
\text { Traumatized IAT [95\% } \\
\text { CIs] }\end{array}$ & $\begin{array}{l}\text { Correlation with Self- } \\
\text { Vulnerable IAT [95\% } \\
\text { CIs] }\end{array}$ \\
\hline Age & $49.44(13.59,19-79)$ & $-0.18^{* *}[-0.29,-0.06]$ & $-0.07[-0.20,0.04]$ \\
PTCI Total & $89.09(38.70,33-203)$ & $0.42^{* * *}[0.31,0.52]$ & $0.30^{* * *}[0.19,0.41]$ \\
PTCI Self & $50.20(25.43,21-138)$ & $0.44^{* * *}[0.32,0.54]$ & $0.31^{* * *}[0.21,0.42]$ \\
PTCI Self-blame & $13.37(7.86,5-35)$ & $0.26^{* * *}[0.14,0.38]$ & $0.19^{* *}[0.06,0.30]$ \\
PTCI World & $25.51(10.48,7-49)$ & $0.30^{* * *}[0.19,0.40]$ & $0.21^{* * *}[0.10,0.33]$ \\
PCL-5 Total & $18.76(16.57,0-69)$ & $0.38^{* * *}[0.28,0.48]$ & $0.34^{* * *}[0.23,0.44]$ \\
DASS Depression & $5.27(5.27,0-21)$ & $0.43^{* * *}[0.32,0.53]$ & $0.35^{* * *}[0.24,0.46]$ \\
DASS Anxiety & $3.06(3.56,0-17)$ & $0.33^{* * *}[0.22,0.43]$ & $0.25^{* * *}[0.13,0.35]$ \\
DASS Stress & $6.39(5.09,0-20)$ & $0.38^{* * *}[0.27,0.47]$ & $0.29^{* * *}[0.18,0.40]$ \\
STAI-Trait & $42.59(13.41,20-80)$ & $0.41^{* * *}[0.29,0.50]$ & $0.30^{* * *}[0.18,0.41]$ \\
STAI-State & $41.65(12.82,20-80)$ & $0.37^{* * *}[0.26,0.47]$ & $0.27^{* * *}[0.15,0.37]$ \\
IATs & & & \\
Self-Traumatized & $-0.27(0.34,-1.10-1.00)$ & - & $0.45^{* * *}[0.34,0.55]$ \\
Self-Vulnerable & $-0.18(0.34,-1.02-0.83)$ & $0.45^{* * *}[0.34,0.55]$ & - \\
Negative event characteristics & & $-0.01[-0.14,0.11]$ & $0.05[-0.07,0.16]$ \\
Distress Then & $9.02(1.46,1-10)$ & $0.28^{* * *}[0.17,0.38]$ & $0.23^{* * *}[0.13,0.34]$ \\
Distress Now & $4.74(2.67,1-10)$ & $0.01[-0.11,0.13]$ & $-0.05[-0.16,0.07]$ \\
Time since event (months) & $189.57(177.21,1-840)$ & \\
\hline
\end{tabular}

PTCI Total/ Self/ Self-blame/ World = Post-Traumatic Cognitions Inventory total score / self subscale score / self-blame subscale score/ world subscale score; PCL-5 Total=Posttraumatic Stress Disorder Checklist for DSM 5; DASS Depression/ Anxiety/ Stress = Depression Anxiety Stress Scales - Short Form Depression subscale/ Anxiety subscale/ Stress subscale; STAI-Trait/ -State $=$ State Trait Anxiety Inventory Trait scale / State scale; IAT $=$ Implicit Association Test. $* p<.05 . * * p<.01 . * * * p<.001$

\begin{tabular}{|c|c|c|c|c|c|c|}
\hline & $B$ [95\% CIs] & $\mathrm{SE}_{\mathrm{B}}$ & $\beta[95 \% \mathrm{CIs}]$ & $\mathrm{SE}_{\beta}$ & $t$ & $p$ \\
\hline Step 1 & $R_{a d j}^{2}=0.18$ & & & & & \\
\hline IAT-ST & $13.92[8.13,19.35]$ & 2.93 & $0.29[0.17,0.41]$ & 0.06 & 4.75 & $<.001$ \\
\hline IAT-SV & $10.06[4.63,15.59]$ & 2.96 & $0.21[0.09,0.33]$ & 0.06 & 3.40 & .001 \\
\hline Step 2 & $\Delta R_{a d j}^{2}=0.66$ & & & & & \\
\hline IAT-ST & $-0.02[-3.73,3.96]$ & 2.01 & $0.00[-0.08,0.08]$ & 0.04 & -0.01 & .993 \\
\hline IAT-SV & $4.56[0.34,8.82]$ & 1.92 & $0.09[0.02,0.17]$ & 0.04 & 2.38 & .018 \\
\hline PTCI Self & $0.51[0.46,0.56]$ & 0.03 & $0.78[0.70,0.86]$ & 0.04 & 19.90 & $<.001$ \\
\hline Step 3 & $\Delta R_{a d j}^{2}=0.68$ & & & & & \\
\hline IAT-ST & $0.43[-3.32,4.23]$ & 1.96 & $0.01[-0.07,0.09]$ & 0.04 & 0.22 & .827 \\
\hline IAT-SV & $4.26[0.15,8.46]$ & 1.87 & $0.09[0.01,0.16]$ & 0.04 & 2.27 & .024 \\
\hline PTCI-Self & $0.50[0.45,0.54]$ & 0.03 & $0.76[0.69,0.84]$ & 0.04 & 19.72 & $<.001$ \\
\hline Gender & $1.40[-0.97,3.64]$ & 1.22 & $0.04[-0.03,0.11]$ & 0.03 & 1.15 & .251 \\
\hline $\begin{array}{l}\text { Distress at time } \\
\text { of event }\end{array}$ & $1.45[0.71,2.22]$ & 0.39 & $0.13[0.06,0.19]$ & 0.03 & 3.72 & $<.001$ \\
\hline
\end{tabular}

IAT-ST = Self-Traumatized IAT; IAT-SV = Self-Vulnerable IAT; PTCI Self=Post-traumatic Cognitions Inventory - Self subscale. Confidence intervals for non-standardized $B$ coefficients are bootstrapped
Table 2 Regression with scores on the PCL-5 as outcome variable that both IATs predicted PCL score, but including PTCISelf score as an additional predictor rendered the SelfTraumatized IAT no longer statistically significant. The
Self-Vulnerable IAT remained a statistically significant predictor of PCL score when further including gender and 
distress at the time of the distressing event as predictors (see Table 2 for details).

\section{Discussion}

This study investigated two different implicit associations tests (IATs) as potential indirect measures of post-traumatic stress disorder (PTSD)-relevant cognitions. Adults who reported having experienced a distressing negative life event completed the two IATs online, alongside self-report questionnaire measures. As a preliminary step, we examined the relationship between scores on the IATs and an established self-report measure of PTSD-relevant dysfunctional cognitions about the self. We then examined the relationship between IAT scores and self-reported post-traumatic stress symptoms related to the distressing event. Scores on both the 'Self-Traumatized' IAT, examining self-traumatized associations, and the 'Self-Vulnerable' IAT, examining selfvulnerable associations, predicted unique variance in selfreported dysfunctional cognitions about the self. Further, scores on both IATs predicted variance in post-traumatic stress symptoms, indicating that stronger self-traumatized and self-vulnerable associations were associated with higher levels of post-traumatic stress symptoms. However, only the Self-Vulnerable IAT explained unique variance in posttraumatic stress symptoms after controlling for self-reported dysfunctional cognitions about the self. Overall, the results provide additional insights into the different components of dysfunctional appraisals in the context of posttraumatic stress symptoms, and highlight the validity and potential utility of these IATs as indirect measures of PTSD-relevant cognitions.

The Self-Traumatized IAT had been previously used in a study with a similar setup to the current one, in that participants came from a community sample who reported having experienced a distressing life event, and completed the study online (Lindgren et al., 2013). In this previous study, scores on the IAT showed unique associations with PTSD symptoms even after controlling for self-report trauma cognitions using the self-subscale of the PTCI. Their study and ours included different measures of PTSD symptoms and different indices of trauma exposure severity. However, these differences seem unlikely in themselves to account for the different results between the two studies. One potential explanation might be differences in the samples: Which (idiosyncratic) dysfunctional appraisals are relevant, and the exact relationship between automatic and reflective processing, could vary according to trauma type (McNally \& Woud, 2019; Woud et al., 2017), and thus sampling differences could easily lead to differences in the relationships between dysfunctional appraisals and trauma symptoms. Our sample was on average substantially older than those in the studies reported by Lindgren et al. (2013), and it may be that there were also differences in type of trauma exposure between the samples. However, this is difficult to compare directly as we did not collect information on multiple trauma exposure. ${ }^{3}$ The relevance of these factors would need following up in a study with a larger sample in order to draw firm conclusions.

The Self-Vulnerable IAT in the current study had previously been used in a sample of Dutch soldiers before and after being deployed to Iraq (Engelhard et al., 2007). When administered 5 months after returning home, scores on this IAT had shown an association with PTSD symptoms at that time, including when controlling for explicitly-reported self-vulnerability. In the current study, the negative events were generally much further in the past and likely much less severe than in the study by Engelhard et al. (2007). However, we also found a cross-sectional association between scores on the Self-Vulnerable IAT and trauma symptoms, despite the variation between studies in the precise measures used. Hence, unlike the Self-Traumatized IAT, the Self-Vulnerable IAT appeared to be measuring something distinct to that measured by the PTCI Self subscale, potentially reflecting an automatic process conferring vulnerability to post-traumatic stress, and which may be less sample-dependent. However, compared to the PTCI, the relationship between scores on the Self-Vulnerable IAT and PCL-5 was relatively weak.

There are a number of other potential reasons why we found the IATs, and the Self-Traumatized IAT in particular, to be weaker predictors of post-traumatic stress symptoms than the PTCI. One possible explanation is that the relatively weak reliability of the IATs compared to the PTCI (perhaps particularly when administered online) reduces their ability to explain additional variance. Further, even the self-subscale of the PTCI asks about a range of different dysfunctional cognitions, whereas the IATs we used (based on previous studies) had a small stimuli set and thus probed a relatively limited set of self-trauma associations. With regard to the Self-Traumatized IAT in particular, it is possible that this is essentially providing a measure of the same cognitive construct as the PTCI, albeit more weakly. That is, although one is an indirect measure and the other explicit, the implicit appraisals measured by the IAT could be readily accessible to conscious introspection and thus could also be detected via an explicit measure. This post-hoc explanation

\footnotetext{
3 Another difference between our study and Study 2 of Lindgren et al. (2013) is that we included two IATs and included scores for both of these in our regression predicting PTSD symptoms. However, we note that even if we repeat the regressions in Table 2 including only one IAT at a time, and further even if we restrict the sample to only those participants who completing the Self-Traumatized IAT first, scores on this IAT do not predict scores on the PCL-5 once PTCI scores are included. Hence this difference between the studies and analyses used seems unlikely to account for the differences in our results.
} 
might help explain the magnitude of the correlation between the IATs and the questionnaire measures, as such high correlations between explicit and implicit measures are often not found and are particularly notable given the internet administration.

However, these results should also be interpreted within the context of broader criticisms of dual system models (e.g., Gladwin \& Figner, 2014; Keren \& Schul, 2009) and IATs in particular as measures of supposedly automatic processes. While the distinction between automatic versus reflective systems may be useful at a holistic level of description, the underlying processes cannot necessarily be neatly divided into reflective ones and automatic ones. Highly overlapping sets of processes could result in emergent behaviour with different degrees of reflectivity, i.e., the amount of evaluation preceding final response selection. From this perspective, attempts to separate reflective and automatic processes would be difficult. In relation to the IAT itself, there is an open and ongoing debate as to what the IAT actually measures, how its results should be interpreted, and which factors may influence its results (e.g., contextual and administration elements). It is beyond the scope of this paper to include an in-depth discussion of these issues, but they are well-documented within the literature (e.g., Greenwald et al., 2020).

Overall, the results suggest that the two IATs measure distinct constructs from each other and both potentially have utility in exploring different aspects of dysfunctional cognitive processes in relation to PTSD. In the case of the SelfTraumatized IAT, if this does not explain additional variance in PTSD symptoms, the question remains of whether it has any utility in the study of PTSD and related appraisals. To fully address this question, research would have to assess whether there are any dissociations between potentially implicit appraisals, as measured on the IAT, and explicit appraisals, as measured by the PTCI, in other contexts, for example in predicting treatment response, longitudinal prediction of symptoms or recovery, or in response to treatments. For example, one experimental study, investigating the effects of a single session of a cognitive bias modification paradigm (CBM) targeting dysfunctional trauma-related appraisals in the context of negative autobiographical life events, found effects on explicit dysfunctional appraisals (as measured by the PTCI) but not on the Self-Traumatized IAT (Woud et al., 2018), and a clinical trial found a similar result (Woud et al., 2021). These results may also reflect a mismatch between the kinds of appraisals trained in the CBM paradigm and the particular associations measured by an IAT with a limited stimuli set. Given the relatively short time needed to complete the IATs, and their adequate level of reliability (even when completed online in uncontrolled circumstances), they would be easy measures to include in PTSD-related projects to explore these questions. The circumstances under which there are dissociations, or not, could provide interesting information about the nature of dysfunctional cognitions in PTSD and help inform our understanding of the potential interplay between automatic and reflective processes in the development of and recovery from PTSD - with the caveat that interpretation of IAT scores is not unproblematic. As a further caveat, in line with the broader IAT literature, even when we found statistically significant relationships between IAT scores and posttraumatic stress symptoms in our regression analyses, these associations were small in magnitude. Developing IATs targeting specific kinds of appraisals (for example, as reflected in the diversity of appraisals in the PTCI and other questionnaire measures of trauma-relevant appraisals, for example about the world being dangerous or fears of going mad), and investigating their relationships with specific PTSD symptom clusters in larger samples may help both in increasing the chances of capturing these dysfunctional processes and providing finer-grained information to inform theory.

A limitation of the study is the reduced sample size compared to the initial one envisaged. Our power analysis and the width of the confidence intervals around our regression estimates indicate that the sample size should be large enough to provide relatively robust estimates of the extent to which scores on the IATs can explain variance in PTSD symptoms above that of the PTCI. However, it precluded the possibility of conducting certain exploratory analyses examining e.g. subgroups of trauma types. The online format of the study also prevents verification of the nature and severity of the negative life event experienced, for example how many of these would fulfil criterion A for a PTSD diagnosis. The relatively large proportion of the sample (55.56\%) selecting 'other' as a trauma type indicates that this was not a typical traumatized sample, and only about $20 \%$ met the cut-off for possible PTSD on the PCL-5. As a reference point, in one study using a representative German sample $(N=2426)$, approximately $10 \%$ of those who reported having experienced a traumatic event met criteria for PTSD (Maercker et al., 2008). In this context, the proportion of the participants meeting the questionnaire cut-off for possible PTSD in our study does not seem especially low. However, it would be important to investigate the IATs in a sample for whom there was a clear traumatic event fitting DSM-5 criteria and in clinical samples diagnosed with PTSD. Research panels as used in this study also risk not being representative of the broader population, and participants' responses to questionnaires may be influenced by familiarity with the same or similar questionnaires from other studies (Göritz, 2007). For example, the high correlations between the IATs and scores on the PTCI and other questionnaires could reflect a sample who are highly compliant and motivated to contribute to psychology research and thus respond relatively accurately on both reaction time and questionnaire measures, reducing the noise in the data. Hence it would 
be useful to replicate these results in a broader population sample. Further, it would be preferable in future studies to allow participants to indicate whether they had experienced multiple traumatic events, which was not the case in the current study. Finally, although the study was pre-registered, the pre-registered analysis plan was not highly specified. However, it seems unlikely that conducting the analyses differently within the specified constraints would lead to different conclusions.

In conclusion, the current study provides further information about the relationship of automatic associations between the self and being traumatized, or vulnerable, with explicitly reported dysfunctional trauma-relevant cognitions and posttraumatic stress symptoms amongst people who have experienced a distressing negative life event. It suggests that these associations have differential relationships between trauma-relevant cognitions and symptoms, and indicates a close relationship between automatic and explicit trauma appraisals in the context of a negative life event. Together, this suggests that the use of the IATs employed in these studies across a broader range of study types and populations could help further probe the nature of the cognitive processes contributing to the development and maintenance of PTSD.

Acknowledgements We would like to thank Christian Leson for setting up and maintaining the server running the IAT software, Lorika Shkreli for her help with data aggregation, Anna Lena Dieberg and Rebecca Raudis for help with the study preparation, Meinald Thielsch for his help with putting the study on the PsyWeb server, and all the participants in the participant pool.

Availability of data, material, and code Data, materials (with the exception of standardized questionnaire measures), analysis scripts and preprint are available on the Open Science Framework at https://osf.io/ qsyu6/.

Funding Open Access funding enabled and organized by ProjektDEAL. Marcella L. Woud is supported by a grant from the Deutsche Forschungsgemeinschaft (DFG; Emmy Noether grant; WO 2018/3-1).

\section{Declarations}

Conflict of Interest We have no known conflict of interest to disclose.

Ethics Approval The study was approved by the Ethics Committee of the Faculty of Psychology, Ruhr-Universität Bochum (approval no. 256).

Consent to Participate All participants in the study provided informed consent.

Open Access This article is licensed under a Creative Commons Attribution 4.0 International License, which permits use, sharing, adaptation, distribution and reproduction in any medium or format, as long as you give appropriate credit to the original author(s) and the source, provide a link to the Creative Commons licence, and indicate if changes were made. The images or other third party material in this article are included in the article's Creative Commons licence, unless indicated otherwise in a credit line to the material. If material is not included in the article's Creative Commons licence and your intended use is not permitted by statutory regulation or exceeds the permitted use, you will need to obtain permission directly from the copyright holder. To view a copy of this licence, visit http://creativecommons.org/licenses/by/4.0/.

\section{References}

American Psychiatric Association. (2013). Diagnostic and statistical manual of mental disorders (5th ed.). American Psychiatric Publishing.

Bluemke, M., Crombach, A., Hecker, T., Schalinski, I., Elbert, T., \& Weierstall, R. (2017). Is the implicit association test for aggressive attitudes a measure for attraction to violence or traumatization? Zeitschrift für Psychologie, 225(1), 54-63. https://doi.org/ 10.1027/2151-2604/a000281

Bockers, E., Roepke, S., Michael, L., Renneberg, B., \& Knaevelsrud, C. (2016). The role of generalized explicit and implicit guilt and shame in interpersonal traumatization and posttraumatic stress disorder. The Journal of Nervous and Mental Disease, 204(2), 95-99. https://doi.org/10.1097/NMD.0000000000000428

Brewin, C. R., Dalgleish, T., \& Joseph, S. (1996). A dual representation theory of posttraumatic stress disorder. Psychological Review, 103(4), 670-686.

Brown, L. A., Belli, G. M., Asnaani, A., \& Foa, E. B. (2019). A review of the role of negative cognitions about oneself, others, and the world in the treatment of PTSD. Cognitive Therapy and Research, 43(1), 143-173. https://doi.org/10.1007/s10608-018-9938-1

Bryant, R. A., \& Guthrie, R. M. (2007). Maladaptive self-appraisals before trauma exposure predict post traumatic stress disorder. Journal of Consulting and Clinincal Psychology, 75(5), 812-815.

Ehlers, A. (1999). Posttraumatische Belastungsstörungen. Hofgrefe.

Ehlers, A., \& Clark, D. M. (2000). A cognitive model of posttraumatic stress disorder. Behaviour Research and Therapy, 38(4), 319-345. https://doi.org/10.1016/S0005-7967(99)00123-0

Ehlers, A., Maercker, A., \& Boos, A. (2000). Posttraumatic stress disorder following political imprisonment: The role of mental defeat, alienation, and perceived permanent change. Journal of Abnormal Psychology, 109(1), 45-55.

Ehring, T., Ehlers, A., \& Glucksman, E. (2008). Do cognitive models help in predicting the severity of posttraumatic stress disorder, phobia, and depression after motor vehicle accidents? A prospective longitudinal study. Journal of Consulting and Clinical Psychology, 76(2), 219-230. https://doi.org/10.1037/0022-006X. 76.2.219

Engelhard, I. M., Huijding, J., van den Hout, M. A., \& de Jong, P. J. (2007). Vulnerability associations and symptoms of posttraumatic stress disorder in soldiers deployed to Iraq. Behaviour Research and Therapy, 45(10), 2317-2325. https://doi.org/10. 1016/j.brat.2007.04.005

Faul, F., Erdfelder, E., Lang, A.-G., \& Buchner, A. (2007). G*Power 3: A flexible statistical power analysis program for the social, behavioral, and biomedical sciences. Behavior Research Methods, 39, 175-191. https://doi.org/10.3758/BF03193146

Foa, E. B., Ehlers, A., Clark, D. M., Tolin, D. F., \& Orsillo, S. M. (1999). The Posttraumatic Cognitions Inventory (PTCI): Development and validation. Psychological Assessment, 11(3), 303-314.

Gladwin, T. E., \& Figner, B. (2014). "Hot" cognition and dual systems: Introduction, criticisms, and ways forward. In E. A. Wilhelms \& V. F. Reyna (Eds.), Neuroeconomics, judgment, and decision making. Psychology Press. 
Göritz, A. S. (2007). Using online panels in psychological research. In The Oxford handbook of internet psychology. Edited by: Joinson, A. N., McKenna, K. Y.A., Postmes, T. and Reips, U.-D. (pp. 473-485). Oxford University Press.

Greenwald, A. G., McGhee, D. E., \& Schwartz, J. L. (1998). Measuring individual differences in implicit cognition: The implicit association test. Journal of Personality and Social Psychology, 74(6), 1464-1480.

Greenwald, A. G., Nosek, B. A., \& Banaji, M. R. (2003). Understanding and using the implicit association test: I. An Improved Scoring Algorithm. Journal of Personality and Social Psychology, 85, 197-216. https://doi.org/10.1037/0022-3514.85.2.197

Greenwald, A. G., Brendl, M., Cai, H., Cvencek, D., Dovidio, J. F., Friese, M., Hahn, A., Hehman, E., Hofmann, W., Hughes, S., Hussey, I., Jordan, C. H., Jost, J., Kirby, T. A., Lai, C. K., Lang, J. W. B., Lindgren, K. P., Maison, D., Ostafin, B., ... Wiers, R. (2020). The implicit association test at age 20: What is known and what is not known about implicit bias. PsyArXiv. https://doi.org/ 10.31234/osf.io/bf97c.

Holmes, E. A., \& Steel, C. (2004). Schizotypy: A vulnerability factor for traumatic intrusions. Journal of Nervous and Mental Disease, 192(1), 28-34. https://doi.org/10.1097/01.nmd.0000105997. $28152.4 \mathrm{~b}$

Keren, G., \& Schul, Y. (2009). Two is not always better than one: A critical evaluation of two-system theories. Perspectives on Psychological Science, 4, 533-550.

Kessler, R. C., Sonnega, A., Bromet, E., Hughes, M., \& Nelson, C. B. (1995). Posttraumatic stress disorder in the National Comorbidity Survey. Archives of General Psychiatry, 52(12), 1048-1060.

Kleim, B., Ehlers, A., \& Glucksman, E. (2012). Investigating cognitive pathways to psychopathology: Predicting depression and posttraumatic stress disorder from early responses after assault. Psychological Trauma: Theory, Research, Practice and Policy, 4(5), 527-537. https://doi.org/10.1037/a0027006

Kleim, B., Grey, N., Wild, J., Nussbeck, F. W., Stott, R., Hackmann, A., Clark, D. M., \& Ehlers, A. (2013). Cognitive change predicts symptom reduction with cognitive therapy for posttraumatic stress disorder. Journal of Consulting and Clinical Psychology, 81(3), 383-393. https://doi.org/10.1037/a0031290

Krüger-Gottschalk, A., Knaevelsrud, C., Rau, H., Dyer, A., Schäfer, I., Schellong, J., \& Ehring, T. (2017). The German version of the posttraumatic stress disorder checklist for DSM-5 (PCL-5): Psychometric properties and diagnostic utility. BMC Psychiatry, 17(1), 379. https://doi.org/10.1186/s12888-017-1541-6

Laux, L., Glanzmann, P., Schaffner, P., \& Spielberger, C. D. (1983). Das state-trait-Angstinventar (STAI). Theoretische Grundlagen und Handanweisung.

Lindgren, K. P., Kaysen, D., Werntz, A. J., Gasser, M. L., \& Teachman, B. A. (2013). Wounds that can't be seen: Implicit trauma associations predict posttraumatic stress disorder symptoms. Journal of Behavior Therapy and Experimental Psychiatry, 44(4), 368-375. https://doi.org/10.1016/j.jbtep.2013.03.003

Maercker, A., Forstmeier, S., Wagner, B., Glaesmer, H., \& Brähler, E. (2008). Posttraumatische Belastungsstörungen in Deutschland. Der Nervenarzt, 79(5), 577. https://doi.org/10.1007/ s00115-008-2467-5

McNally, R. J., \& Woud, M. L. (2019). Innovations in the study of appraisals and PTSD: A commentary. Cognitive Therapy and Research, 43(1), 295-302. https://doi.org/10.1007/ s10608-018-09995-2

Müller, J., Wessa, M., Rabe, S., Dörfel, D., Knaevelsrud, C., Flor, H., Maercker, A., \& Karl, A. (2010). Psychometric properties of the
Posttraumatic Cognitions Inventory (PTCI) in a German sample of individuals with a history of trauma. Psychological Trauma: Theory, Research, Practice, and Policy, 2(2), 116-125. https:// doi.org/10.1037/a0018603

National Institute for Health and Clinical Excellence. (2018). Posttraumatic stress disorder (NICE guideline 116). National Institute for Health and Clinical Excellence. https://www.nice.org.uk/guida nce/ng116

Nilges, P., \& Essau, C. (2015). Die Depressions-Angst-Stress-Skalen: Der DASS - ein Screeningverfahren nicht nur für Schmerzpatienten. Der Schmerz, 29(6), 649-657. https://doi.org/10.1007/ s00482-015-0019-z

RStudio, Inc. (2016). RStudio: Integrated Development for R. http:// www.rstudio.com/

Rüsch, N., Schulz, D., Valerius, G., Steil, R., Bohus, M., \& Schmahl, C. (2011). Disgust and implicit self-concept in women with borderline personality disorder and posttraumatic stress disorder. European Archives of Psychiatry and Clinical Neuroscience, 261(5), 369-376. https://doi.org/10.1007/s00406-010-0174-2

Teachman, B. A., Clerkin, E. M., Cunningham, W. A., Dreyer-Oren, S., \& Werntz, A. (2019). Implicit cognition and psychopathology: Looking back and looking forward. Annual Review of Clinical Psychology, 15, 123-148. https://doi.org/10.1146/annurev-clinp sy-050718-095718

Vossbeck-Elsebusch, A. N., Freisfeld, C., \& Ehring, T. (2014). Predictors of posttraumatic stress symptoms following childbirth. BMC Psychiatry, 14, 200. https://doi.org/10.1186/1471-244X-14-200

Weathers, F. W., Litz, B. T., Keane, T. M., Palmieri, P. A., Marx, B. P., \& Schnurr, P. P. (2013). The PTSD Checklist for DSM-5 (PCL-5). www.ptsd.va.gov

Woud, M. L., Holmes, E. A., Postma, P., Dalgleish, T., \& Mackintosh, B. (2012). Ameliorating intrusive memories of distressing experiences using computerized reappraisal training. Emotion, 12(4), 778-784. https://doi.org/10.1037/a0024992

Woud, M. L., Verwoerd, J., \& Krans, J. (2017). Modification of cognitive biases related to posttraumatic stress: A systematic review and research agenda. Clinical Psychology Review, 54, 81-95. https:// doi.org/10.1016/j.cpr.2017.04.003

Woud, M. L., Zlomuzica, A., Cwik, J. C., Margraf, J., Shkreli, L., Blackwell, S. E., Gladwin, T. E., \& Ehring, T. (2018). Effects of appraisal training on responses to a distressing autobiographical event. Journal of Anxiety Disorders. https://doi.org/10.1016/j. janxdis.2018.03.010

Woud, M. L., Cwik, J. C., de Kleine, R. A., Blackwell, S. E., Würtz, F., \& Margraf, J. (2019a). Assessing trauma-related appraisals by means of a scenario-based approach. Cognitive Therapy and Research, 43, 185-198. https://doi.org/10.1007/ s10608-018-9956-z

Woud, M. L., Kleim, B., \& Cwik, J. C. (2019b). Editorial for the special issue on negative appraisals in trauma: Current status and future directions for research. Cognitive Therapy and Research, 43(1), 139-142. https://doi.org/10.1007/s10608-018-09992-5

Woud, M. L., Blackwell, S. E., Shkreli, L., Würtz, F., Cwik, J. C., Margraf, J., Holmes, E. A., Steudte-Schmiedgen, S., Herpertz, S., \& Kessler, H. (2021). The effects of modifying dysfunctional appraisals in posttraumatic stress disorder using a form of cognitive bias modification: Results of a randomized controlled trial in an inpatient setting. Psychotherapy and Psychosomatics, 90, 386-482. https://doi.org/10.1159/000514166

Publisher's Note Springer Nature remains neutral with regard to jurisdictional claims in published maps and institutional affiliations. 